PREPARED FOR THE U.S. DEPARTMENT OF ENERGY, UNDER CONTRACT DE-AC02-76CH03073

PPPL-3569

PPPL-3569

UC-70

Nonlinear gyroviscous force in a collisionless plasma

E.V. Belova

May 2001

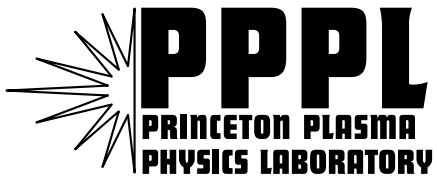

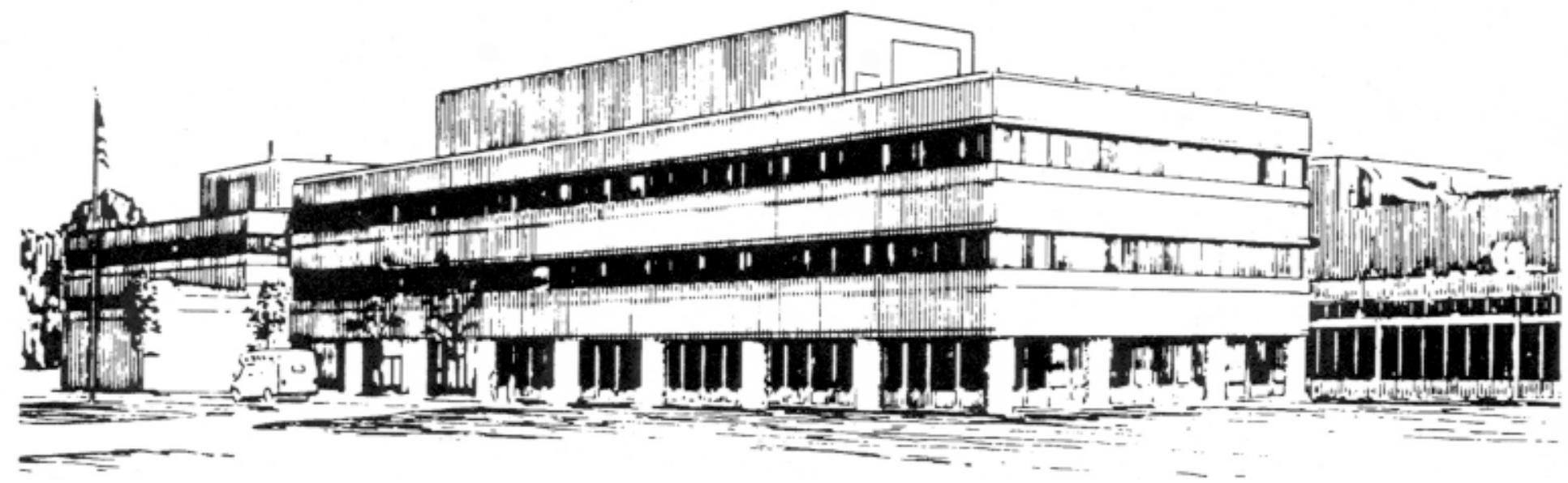

PRINCETON PLASMA PHYSICS LABORATORY PRINCETON UNIVERSITY, PRINCETON, NEW JERSEY 


\section{PPPL Reports Disclaimer}

This report was prepared as an account of work sponsored by an agency of the United States Government. Neither the United States Government nor any agency thereof, nor any of their employees, makes any warranty, express or implied, or assumes any legal liability or responsibility for the accuracy, completeness, or usefulness of any information, apparatus, product, or process disclosed, or represents that its use would not infringe privately owned rights. Reference herein to any specific commercial product, process, or service by trade name, trademark, manufacturer, or otherwise, does not necessarily constitute or imply its endorsement, recommendation, or favoring by the United States Government or any agency thereof. The views and opinions of authors expressed herein do not necessarily state or reflect those of the United States Government or any agency thereof.

\section{Availability}

This report is posted on the U.S. Department of Energy's Princeton Plasma Physics Laboratory Publications and Reports web site in Calendar Year 2001. The home page for PPPL Reports and Publications is: http://www.pppl.gov/pub_report/

DOE and DOE Contractors can obtain copies of this report from:

U.S. Department of Energy

Office of Scientific and Technical Information

DOE Technical Information Services (DTIS)

P.O. Box 62

Oak Ridge, TN 37831

Telephone: (865) 576-8401

Fax: (865) 576-5728

Email: reports@adonis.osti.gov

This report is available to the general public from:

National Technical Information Service

U.S. Department of Commerce

5285 Port Royal Road

Springfield, VA 22161

Telephone: 1-800-553-6847 or

(703) 605-6000

Fax: (703) 321-8547

Internet: http://www.ntis.gov/ordering.htm 


\title{
Nonlinear gyroviscous force in a collisionless plasma
}

\author{
E. V. Belova \\ Princeton Plasma Physics Laboratory, Princeton, NJ 08543, USA
}

Nonlinear gyroviscous forces in a collisionless plasma with temperature variations are calculated from the gyrofluid moments of the gyrokinetic Vlasov equation. The low-frequency gyrokinetic ordering is assumed, and an additional finite Larmor radius (FLR) expansion in a parameter $\epsilon_{\perp} \equiv\left(k_{\perp} \rho\right)^{2}<1$ is performed. This approach leads naturally to an expression for the gyroviscous force, $\left(\nabla \cdot \pi_{\mathrm{g}}\right)$, in terms of the gyrocenter distribution function, thus including all resonant effects, and represents a systematic FLR expansion in a general form (no assumption of any closure is made). The obtained results can be used, in particular, in kinetic calculations, and allow the inclusion of FLR corrections into the usual drift-kinetic formulation via particle stress tensor. The expression of $\left(\nabla \cdot \boldsymbol{\pi}_{\mathbf{g}}\right)$ is also calculated in terms of the particle-fluid moments by making the transformation from the gyrocenter to particle coordinates. The calculated $\left(\nabla \cdot \boldsymbol{\pi}_{\mathbf{g}}\right)$ represents a modification of the Braginskii gyroviscosity for a collisionless plasma with $\nabla T \neq 0$. It is compared with previous calculations based on the traditional fluid approach. As a byproduct of the gyroviscosity calculations, we derive a set of nonlinear reduced gyrofluid (and a corresponding set of particle-fluid) moment equations with FLR corrections. The equations exhibit a generalized form of the "gyroviscous cancellation", which appears in an arbitrary high moment equation of an infinite set of fluid equations.

PACS numbers: 52.65.Tt, 52.25.Dg, 52.65.Kj 


\section{INTRODUCTION}

For many problems arising in space and laboratory plasmas, the thermal Larmor radius of a particle species (ions or electrons) is small compared to the equilibrium scale lengths, as well as the perpendicular (with respect to the background magnetic field) wavelengths of interest. Simplified physical models are often used to describe the low-frequency phenomena in such a plasma. A magnetohydrodynamic (MHD) fluid description or a standard guiding-center (drift-kinetic) formulation represent the lowest order approximations for collisional and collisionless plasmas, respectively. When finite Larmor radius (FLR) effects are important, corrections can be introduced in these models in a perturbative way by making an expansion with respect to the small parameter $\epsilon \sim \rho / L \sim \omega / \omega_{c}$, where $\rho / L$ is the ratio of the Larmor radius to the perpendicular scale length, and $\omega_{c}$ is the cyclotron frequency.

There are two different approaches to the derivation of FLR fluid equations. In the first and more traditional approach ${ }^{1}$, a set of FLR equations is derived directly via a systematic expansion of the hierarchy of fluid moments of Vlasov equation. When, in addition, a closure relation (collisional, for example) is assumed, a closed set of magnetohydrodynamic, FLRcorrected, fluid equations can be obtained ${ }^{1-3}$.

The second approach is based on a set of nonlinear gyrokinetic equations ${ }^{4-6}$, derived assuming the gyrokinetic ordering:

$$
\epsilon=\frac{\rho}{L_{n}} \sim \frac{\omega}{\omega_{c}} \sim \frac{k_{\|}}{k_{\perp}} \ll 1 ; \quad \epsilon_{\delta}=\frac{\delta F}{F_{0}} \sim \frac{e \varphi}{T} \ll 1 ; \quad \epsilon_{\perp}=\left(k_{\perp} \rho\right)^{2} \sim 1
$$

Here $L_{n}$ is the equilibrium density gradient scale length, $k_{\|}$and $k_{\perp}$ are the parallel and perpendicular wave numbers, $\varphi$ is the perturbed electrostatic potential, $\delta F$ and $F_{0}$ are the perturbed and equilibrium distribution functions, and $\epsilon \sim \epsilon_{\delta}$ are the expansion parameters. 
In the second approach, a set of gyrofluid equations ${ }^{7-9}$ is obtained by taking moments of the gyrokinetic Vlasov equation. As a result, these equations are formulated in terms of the moments of the gyrocenter distribution function, rather than the particle distribution function. The gyrocenter-fluid (GF) moments can be expressed in terms of the particle-fluid (PF) moments, producing a set of nonlinear reduced fluid equations with FLR corrections. The gyrofluid formulation greatly simplifies the derivation of the reduced FLR fluid equations as compared to the usual fluid moment approach. This simplification comes from the reduced dimensionality of the gyrokinetic Vlasov equation (no gyroangle dependence), in which the expansion with respect to the small parameter $\epsilon \sim \epsilon_{\delta}$ has already been made. An additional advantage of the gyrofluid approach is the separation of the equilibrium and perturbed fields, which allows different scale lengths for the background fields and the perturbation. Thus, it is valid for $\left(k_{\perp} \rho\right) \sim 1$, however, in practice, an expansion in $\epsilon_{\perp}=\left(k_{\perp} \rho\right)^{2}$ can be made to facilitate the transformation between the gyrocenter-fluid and particle-fluid moments.

In this paper, we derive the FLR corrections to the particle stress tensor for a collisionless plasma using the gyrokinetic formulation. The goal of this calculation is twofold. First, the FLR corrected stress tensor expressed in terms of the gyrocenter distribution function can be used to include the higher order FLR corrections into the drift-kinetic model. It can be used directly or as a part of the ion perpendicular current calculation in the numerical/analytical schemes, which rely on the so-called particle $\operatorname{closure}^{10,11}$. For $k_{\perp} \rho \ll 1$, such a scheme can be considerably cheaper and more efficient than a gyrokinetic one. Second, the particlefluid gyroviscosity, $\boldsymbol{\pi}_{g}$, is obtained by expressing the gyrocenter-fluid moments in terms of the particle-fluid moments. The fluid expression so obtained yields a useful benchmark for comparison with similar calculations based on the standard fluid approach ${ }^{2,3,12}$. The 
calculated gyroviscous force allows one to incorporate FLR (diamagnetic) effects into a fluid description of plasma.

It is well known that in the fluid description, in the limit of small but finite Larmor radius, the FLR corrections can be included in the momentum equation in the form of the gyroviscous stress tensor ${ }^{1}$. The Braginskii fluid equations were derived assuming collisional ordering, and neglect the contribution of the higher-order fluid moments (heat fluxes, in particular) into the gyroviscosity. It has recently been demonstrated ${ }^{3}$ that in a plasma with temperature variations, the contributions to the gyroviscous stresses from the gradients of the heat flux are comparable to that of the velocity gradients. The self-consistent inclusion of the heat fluxes is also important for the so-called gyroviscous cancellation ${ }^{2,3,12}$, when $T \neq$ const. (The gyroviscous cancellation is usually understood as the cancellation of the convective derivative associated with the diamagnetic velocity from the total time derivative: $d^{\prime} / d t=d / d t-\left(\mathbf{V}_{*} \cdot \nabla\right)$, when the gyroviscous force is included in the momentum equation.)

In this paper, the expression for the gyroviscous force is obtained in terms of the particle distribution function, and all higher order moments are retained without truncation. Therefore, it represents a systematic FLR expansion in a general form (no assumption of any closure is made), and includes all relevant kinetic effects. In addition, the gyroviscous cancellations are recovered automatically, since the diamagnetic velocity does not appear in the total time derivative in the gyrofluid equations. Results of our calculations are in general agreement with those of $\mathrm{Smolyakov}^{3}$, who used an alternative fluid approach. It is demonstrated, in particular, that the previous discrepancy between calculations based on the fluid theory ${ }^{3}$ and the gyrofluid derivation ${ }^{7}$ is related to the inconsistent treatment of the parallel heat fluxes in Ref. 7. 
This paper is organized as follows. In Sec. II, we present the gyrokinetic Vlasov equation and the relation between the particle phase-space and the gyrokinetic phase-space variables used in this paper. Our calculations are based on the nonlinear gyrokinetic equations ${ }^{4-6}$, and are carried out up to the second order in $\epsilon, \epsilon \delta$, assuming the gyrokinetic ordering Eq. (1). A general gyrofluid equation is derived in Sec. III. A relation between the gyrocenter-fluid moments and the particle-fluid moments, and a general reduced fluid moment equation are also presented. The derivation is greatly simplified by the small Larmor radius assumption, $k_{\perp} \rho \ll 1$, and the expansion in $\epsilon_{\perp}$ is carried out through $O\left(\epsilon_{\perp}\right)$ order. The first six reduced FLR-corrected fluid equations are presented, and these equations are compared with the previous fluid and gyrofluid results. The parallel component of the gyroviscous force is found from the parallel component of the nonlinear FLR-corrected momentum equation. The derivation of the perpendicular component of $\left(\nabla \cdot \pi_{\mathrm{g}}\right)$ is presented in Sec. IV, where two different forms of the gyroviscous force ${ }^{2,12}$ are given and discussed. A summary and conclusions are given in Section V.

\section{GYROKINETIC VLASOV EQUATION}

The gyroviscous forces are calculated assuming a low beta plasma, using the electrostatic approximation, and neglecting the magnetic curvature terms. To simplify notation, we set $e=m_{i}=c=1$ throughout the paper. The gyrokinetic equations used in this paper are derived using the action-variational Lie perturbation method ${ }^{4,13,5}$. The derivation is carried out in two steps. First, the guiding-center equations of motion are derived. This is done by making a transformation from physical space coordinates, $\mathbf{z}=\left(\mathbf{x}, v_{\|}, v_{\perp}^{2} / 2 B, \theta_{0}\right)$, to guidingcenter coordinates $\mathbf{Z}^{\prime}=\left(\mathbf{X}^{\prime}, U^{\prime}, \mu^{\prime}, \theta^{\prime}\right)$ in such a way that the gyroangle dependence in a 
guiding-center Hamiltonian is "transformed away", and the resulting equations of motion are gyroangle independent. The coordinate transformation is the near identity transformation with small parameter being $\epsilon=\rho / L$, and eliminates the gyroangle dependence associated with the background inhomogeneities. When magnetic curvature is neglected, "new" and "old" variables are simply related by: $\mathbf{X}^{\prime}=\mathbf{x}-\boldsymbol{\rho}, U^{\prime}=v_{\|}, \mu^{\prime}=v_{\perp}^{2} / 2 B, \quad \theta^{\prime}=\theta_{0}$.

The gyroangle dependence introduced by the perturbed field is removed by the second transformation from the guiding-center to gyrocenter coordinates: $\mathbf{Z}^{\prime} \rightarrow \mathbf{Z}=(\mathbf{X}, U, \mu, \theta)$. The small parameter $\epsilon_{\delta}$ associated with this transformation is the perturbation amplitude, and the gyrokinetic equations used in this paper are second order accurate in $\epsilon_{\delta}$. In this order, the guiding-center and gyrocenter coordinates are related by:

$$
Z^{j}\left(\mathbf{Z}^{\prime}\right)=Z^{\prime j}+G_{1}^{j}+\frac{1}{2} G_{1}^{i} \frac{\partial G_{1}^{j}}{\partial Z^{\prime i}}+G_{2}^{j}
$$

where $G_{1}^{j}$ and $G_{2}^{j}$ are the components of the generating vector of the first order and second order (in the perturbation amplitude) transformations respectively, given by $(n=1,2)$

$$
\begin{aligned}
\mathbf{G}_{n} & =-\hat{\mathbf{b}} \partial S_{n} / \partial U-\frac{1}{B} \hat{\mathbf{b}} \times \nabla S_{n} \\
G_{n}^{U} & =\nabla_{\|} S_{n} \\
G_{n}^{\mu} & =\partial S_{n} / \partial \theta \\
G_{n}^{\theta} & =-\partial S_{n} / \partial \mu
\end{aligned}
$$

The equations for the scalar functions $S_{1}$ and $S_{2}=O\left(\epsilon_{\delta}^{2}\right)$ are determined by the choice of the transformation (2), and are given up to $O\left(\epsilon \epsilon_{\delta}, \epsilon_{\delta}^{2}\right)$ in the Appendix. The lowest order solution for $S_{1}$ is $S_{1}=\tilde{\Psi} / B$, where $\tilde{\Psi}=\int^{\theta} \tilde{\varphi} d \theta$, and $\tilde{\varphi}=\varphi-\langle\varphi\rangle$ is the gyroangle dependent part of the perturbed electrostatic potential $\varphi=\varphi(\mathbf{X}+\boldsymbol{\rho}, t)$.

The gyrocenter distribution function is gyroangle independent, $F=F(\mathbf{X}, U, \mu)$, and satisfies the gyrokinetic Vlasov equation ${ }^{4,5}$ : 


$$
\frac{\partial F}{\partial t}+\left(\hat{\mathbf{b}} U+\frac{1}{B} \hat{\mathbf{b}} \times \nabla\langle\Phi\rangle\right) \cdot \nabla F-\hat{\mathbf{b}} \cdot \nabla\langle\Phi\rangle \frac{\partial F}{\partial U}=0
$$

where $\hat{\mathbf{b}}=\mathbf{B} / B$ and $\Phi=\varphi-\frac{1}{2 B} \partial\left(\tilde{\varphi}^{2}\right) / \partial \mu+\frac{1}{2 B^{2}} \hat{\mathbf{b}} \cdot \nabla \tilde{\Psi} \times \nabla \tilde{\varphi}$, and angular brackets denote the gyroaveraging. We will assume that $\nabla_{\|} F_{0}=0$. Note that the gyrokinetic ordering implies that $\nabla_{\perp} \delta F \sim \nabla_{\perp} F_{0} \sim \epsilon$, where from now on $\epsilon$ will denote both $\epsilon$ and $\epsilon_{\delta}$.

It is possible to directly calculate the stress tensor in terms of the gyrocenter distribution function by using Eq. (2) and making a coordinate transformation in the integral $\mathbf{P}=$ $\int \mathbf{v} \mathbf{v} f d^{3} \mathbf{v}$. However, since we are only interested in the expression for the force, $\nabla \cdot \mathbf{P}$, the calculations can be simplified. Thus in this paper, we are using the gyrofluid approach to derive a set of FLR corrected fluid equations, and the parallel component of the gyroviscous force is obtained from the parallel momentum equation. The perpendicular component of $\nabla \cdot \mathbf{P}$ is calculated by expressing the perpendicular fluid velocity in terms of the gyrofluid moments through $O\left(\epsilon^{2}\right)$ order.

\section{REDUCED FLUID EQUATIONS}

\section{A. Gyrofluid equations}

In the gyrofluid approach ${ }^{7,8}$, a set of coupled nonlinear gyrofluid equations is obtained by taking moments of the gyrokinetic Vlasov equation (3). Due to the ordering (1), the first order $O(\epsilon)$ accurate Vlasov equation and the first order accurate transformation between the gyrocenter-fluid and the particle-fluid moments are sufficient ${ }^{7}$ to derive the fluid equations valid up to $O\left(\epsilon^{2}\right)$. We define the general gyrocenter-fluid (GF) moment as

$$
M_{k l}(\mathbf{X}, t) \equiv\left\|\mu^{k} U^{l}\right\|^{G F}=\int \mu^{k} U^{l} F d \mu d U
$$

Multiplying the gyrokinetic Vlasov equation Eq. (3) by $\mu^{k} U^{l}$, and taking the integral, we 
obtain two equations

for $l$ - even:

$$
\frac{d^{\prime} M_{k l}}{d t}+\nabla_{\|} M_{k l+1}+\frac{1}{2 B^{2}}\left\{\nabla_{\perp}^{2} \varphi, M_{k+1 l}\right\}=0
$$

for $l$ - odd:

$$
\frac{d^{\prime} M_{k l}}{d t}+\nabla_{\|}\left(M_{k l+1}+l M_{k l-1} \varphi\right)+\frac{1}{2 B^{2}}\left\{\nabla_{\perp}^{2} \varphi, M_{k+1 l}\right\}+\frac{l}{2 B} M_{k+1 l-1} \nabla_{\|} \nabla_{\perp}^{2} \varphi=0
$$

where $d^{\prime} / d t \equiv \partial / \partial t+\left(\mathbf{v}_{E} \cdot \nabla\right)$, and $\{f, g\} \equiv \hat{\mathbf{b}} \cdot \nabla f \times \nabla g$. In the derivation, it has been assumed that $M_{k l}=O(1)$ for any $l$ - even, and $M_{k l}=O(\epsilon)$ when $l$ is odd, and terms of order higher than $O\left(\epsilon^{2}\right)$ have been neglected. An expansion with respect to small parameter $\epsilon_{\perp}$ has been made in the gyrokinetic Vlasov equation, so that $\langle\Phi\rangle=J_{0} \varphi+O\left(\epsilon^{2}\right) \approx\left(1+\frac{\mu}{2 B} \nabla_{\perp}^{2}\right) \varphi$, where $J_{0}$ is the Bessel function. Equations (5) and (6) are the gyrofluid equations written in a general form, and valid up to $O\left(\epsilon^{2}\right)$ and $O\left(\epsilon_{\perp}\right)$ order. The equations for GF density $N=M_{00}$, parallel momentum $N V_{\|}=M_{01}$, and the GF perpendicular and parallel pressure, $P_{\perp}=B M_{10}$ and $P_{\|}=M_{02}$ can be obtained from Eqs. (5) and (6). To the same order in $\epsilon_{\perp}$ and with the same assumptions, these equations agree with the gyrofluid equations given by Brizard ${ }^{7}$.

\section{B. Relation between gyrocenter-fluid and particle-fluid moments}

In this Section we derive a relation between the gyrocenter-fluid and particle-fluid moments to first order accuracy in $\epsilon$ and $\epsilon_{\perp}$. Higher-order $O\left(\epsilon^{2}\right)$ terms are not needed for the derivation of the reduced fluid equation, because their contribution in the particle-fluid equations is an order higher than that considered in this paper. We define a general particle-fluid moment as: 


$$
m_{k l}(\mathbf{x}, t) \equiv\left\|\mu^{k} U^{l}\right\|^{P F}=\int\left(v_{\perp}^{2} / 2 B\right)^{k}\left(v_{\|}\right)^{l} f d^{3} \mathbf{v}
$$

In terms of guiding-center distribution function, $F_{g c}$, the PF moment can be written as ${ }^{4}$ :

$$
m_{k l}(\mathbf{x}, t)=\int \mu^{k} U^{l} F_{g c}(\mathbf{Z}) \delta(\mathbf{X}+\boldsymbol{\rho}-\mathbf{x}) d^{6} \mathbf{Z}
$$

where $d^{6} \mathbf{Z}=B d^{3} \mathbf{X} d U d \mu d \theta$. Using the relation between the guiding-center distribution function and the gyrocenter distribution function given in the Appendix, it becomes:

$$
m_{k l}(\mathbf{x}, t)=\int \mu^{k} U^{l}\left[F+\frac{\tilde{\varphi}}{B} \frac{\partial F}{\partial \mu}\right] \delta(\mathbf{X}+\boldsymbol{\rho}-\mathbf{x}) d^{6} \mathbf{Z}
$$

After the Larmor radius expansion, the PF moment in terms of GF moments to first order in $\epsilon$ and $\epsilon_{\perp}$ is:

$$
m_{k l}=M_{k l}+\frac{1}{2 B} \nabla_{\perp}^{2} M_{k+1 l}+\frac{(k+1)}{B^{2}} M_{k l} \nabla_{\perp}^{2} \varphi
$$

For $l$ - odd, the last term on the RHS is $O\left(\epsilon^{2}\right)$, and should be dropped. The difference between $\mathrm{PF}$ and GF moments is $O\left(\epsilon_{\perp}\right)$, and to this order, the inversion of the Eq. (10) is trivial:

$$
M_{k l}=m_{k l}-\frac{1}{2 B} \nabla_{\perp}^{2} m_{k+1 l}-\frac{(k+1)}{B^{2}} m_{k l} \nabla_{\perp}^{2} \varphi
$$

\section{Particle-fluid moment equations}

In this Section the relation between the GF moments and the PF moments, Eq. (10), and the gyrofluid equations Eqs. (5) and (6) are used to derive the evolution equation for PF moment $m_{k l}$. The calculations are carried with $O\left(\epsilon^{2}\right)$ and $O\left(\epsilon_{\perp}\right)$ accuracy. Taking the time derivative $d^{\prime} / d t$ of the PF moment $m_{k l}$, and using the Eqs. (10) and (6), we obtain

$$
\begin{aligned}
\frac{d^{\prime} m_{k l}}{d t}= & -\nabla_{\|}\left(M_{k l+1}+l M_{k l-1} \varphi\right)-\frac{1}{2 B^{2}}\left\{\nabla_{\perp}^{2} \varphi, M_{k+1 l}\right\}-\frac{l}{2 B} M_{k+1 l-1} \nabla_{\|} \nabla_{\perp}^{2} \varphi \\
& +\frac{1}{2 B} \frac{d^{\prime}}{d t} \nabla_{\perp}^{2} M_{k+1 l}+\frac{(k+1)}{B^{2}} M_{k l} \frac{d^{\prime}}{d t} \nabla_{\perp}^{2} \varphi
\end{aligned}
$$


Now we can substitute the GF moments in terms of the PF moments, neglecting $O\left(\epsilon^{3}\right)$ and $O\left(\epsilon_{\perp}^{2}\right)$ terms, and use the commutation relation ${ }^{7} d^{\prime} / d t \nabla_{\perp}^{2}=\nabla_{\perp}^{2} d^{\prime} / d t+\left\{\nabla_{\perp}^{2} \varphi, \quad\right\} / B-$ $2 \nabla_{\perp} \cdot\left\{\nabla_{\perp} \varphi, \quad\right\} / B$ to obtain

for $l$ - even:

$$
\frac{d^{\prime} m_{k l}}{d t}=-\nabla_{\|} m_{k l+1}+m_{k l}(k+1) \frac{d^{\prime}}{d t} \nabla_{\perp}^{2} \varphi / B^{2}-\nabla_{\perp} \cdot\left\{\nabla_{\perp} \varphi, m_{k+1 l}\right\} / B^{2}
$$

for $l$ - odd:

$$
\begin{aligned}
& \frac{d^{\prime} m_{k l}}{d t}=-\nabla_{\|} m_{k l+1}-l m_{k l-1} \nabla_{\|} \varphi-\nabla_{\perp} \cdot\left\{\nabla_{\perp} \varphi, m_{k+1 l}\right\} / B^{2} \\
&+\left[(k+1) m_{k l+1} / B^{2}-l m_{k+1 l-1} / B\right] \nabla_{\|} \nabla_{\perp}^{2} \varphi
\end{aligned}
$$

where $d^{\prime} / d t=\partial / \partial t+\left(\mathbf{v}_{E} \cdot \nabla\right)$. When the equilibrium distribution function is bi-Maxwellian, the last term in the Eq. (13) is proportional to $\left(p_{\|}^{(0)}-p_{\perp}^{(0)}\right)$ and can be neglected if the zero-order pressure anisotropy is small. In this case, the FLR corrections in the odd- $l$ moment equations appear only in the nonlinear $\left(\epsilon_{\delta}^{2}\right)$ term. The equations (12) and (13) represent an infinite set of coupled FLR-corrected reduced fluid equations derived through $O\left(\epsilon^{2}\right)$ and $O\left(\epsilon_{\perp}\right)$ order. Note that these particle-fluid equations exhibit a generalized form of "gyroviscous cancellation" (a cancellation of the $\left(\mathbf{v}_{*} \cdot \nabla\right)$ term from the total time derivative on the LHS).

\section{Example: first six reduced fluid equations}

Using the general form of the particle-fluid equations (12) and (13), the time evolution equations for the first four particle-fluid moments $n=\|1\|^{P F}=m_{00}, \quad n v_{\|}=\|U\|^{P F}=m_{01}$, $p_{\perp}=\|B \mu\|^{P F}=B m_{10}$, and $p_{\|}=\left\|U^{2}\right\|^{P F}=m_{02}$, becomes

$$
\frac{d^{\prime} n}{d t}=-\nabla_{\|}\left(n v_{\|}\right)+\frac{n^{(0)}}{B^{2}} \frac{d^{\prime}}{d t} \nabla_{\perp}^{2} \varphi-\nabla_{\perp} \cdot\left\{\nabla_{\perp} \varphi, p_{\perp}\right\} / B^{3}
$$




$$
\begin{aligned}
\frac{d^{\prime} p_{\perp}}{d t} & =-\nabla_{\|}\|U \mu B\|+\frac{2 p_{\perp}^{(0)}}{B^{2}} \frac{d^{\prime}}{d t} \nabla_{\perp}^{2} \varphi-\nabla_{\perp} \cdot\left\{\nabla_{\perp} \varphi,\left\|\mu^{2}\right\|\right\} / B \\
\frac{d^{\prime} p_{\|}}{d t} & =-\nabla_{\|}\left\|U^{3}\right\|+\frac{p_{\|}^{(0)}}{B^{2}} \frac{d^{\prime}}{d t} \nabla_{\perp}^{2} \varphi-\nabla_{\perp} \cdot\left\{\nabla_{\perp} \varphi,\left\|U^{2} \mu\right\|\right\} / B^{2} \\
n \frac{d^{\prime} v_{\|}}{d t} & =-\nabla_{\|} p_{\|}-n \nabla_{\|} \varphi-\nabla_{\perp} \cdot\left\{\nabla_{\perp} \varphi,\|U \mu\|\right\} / B^{2}+\frac{p_{\|}^{(0)}-p_{\perp}^{(0)}}{B^{2}} \nabla_{\|} \nabla_{\perp}^{2} \varphi
\end{aligned}
$$

These equations are written in terms of the particle-fluid moments, and the index " 0 " is used for the equilibrium quantities. Using the notation of Ref. 7, the third and fourth-order moments can be written as follows:

$$
\begin{aligned}
& \|U \mu\| B=p_{\perp} v_{\|}+q_{\|}^{(\perp)}, \quad\left\|U^{3}\right\|=3 p_{\|} v_{\|}+2 q_{\|}^{(\|)}, \\
& \left\|\mu^{2}\right\| B^{2}=\frac{2 p_{\perp}^{2}}{n}+2 R_{\perp}, \quad\left\|U^{2} \mu\right\| B=\frac{p_{\perp} p_{\|}}{n}+R_{\times} .
\end{aligned}
$$

Here $q_{\|}=q_{\|}^{(\perp)}+q_{\|}^{(\|)}$is the parallel heat flux, and $R_{\perp}$ and $R_{\times}$are the components of the energy-weighted stress tensor $\mathbf{R}$. From the time evolution equations for the third-order moments $m_{11}$ and $m_{03}$, the equations for the components of the parallel heat flux can also be derived

$$
\begin{aligned}
\frac{d^{\prime} q_{\|}^{(\perp)}}{d t}= & -\nabla_{\|}\left\|\mu U^{2}\right\| B+T_{\perp} \nabla_{\|} p_{\|}-\nabla_{\perp} \cdot\left\{\nabla_{\perp} \varphi,\left\|\mu^{2} U\right\| B-T_{\perp}\|\mu U\|\right\} / B^{2} \\
& +\frac{T_{\perp}^{(0)}}{n B^{2}}\left(p_{\|}^{(0)}-p_{\perp}^{(0)}\right) \nabla_{\|} \nabla_{\perp}^{2} \varphi \\
\frac{d^{\prime} q_{\|}^{(\|)}}{d t}= & -\frac{1}{2} \nabla_{\|}\left\|U^{4}\right\|+\frac{3}{2} T_{\|} \nabla_{\|} p_{\|}-\frac{1}{2} \nabla_{\perp} \cdot\left\{\nabla_{\perp} \varphi,\left\|\mu U^{3}\right\|-3 T_{\|}\|\mu U\|\right\} / B^{2}
\end{aligned}
$$

In Eqs. (20) and (21), the equilibrium distribution function, $f^{(0)}$, is assumed to be biMaxwellian, and $T_{\perp}=p_{\perp} / n$ and $T_{\|}=p_{\|} / n$ are the perpendicular and parallel temperatures.

When the FLR corrections are neglected, these equations take a simple form

$$
\begin{aligned}
& \frac{d^{\prime} q_{\|}^{(\perp)}}{d t}=-p_{\|} \nabla_{\|} T_{\perp}-\nabla_{\|} R_{\times} \\
& \frac{d^{\prime} q_{\|}^{(\|)}}{d t}=-\frac{3}{2} p_{\|} \nabla_{\|} T_{\|}-\nabla_{\|} R_{\|}
\end{aligned}
$$


The equations for $\mathbf{R}$ and other higher-order moment equations can be obtained from Eqs. (12) and (13) in the same way. This infinite set of coupled fluid equations cannot in general be closed unless some assumption about the distribution function is made.

It is worth-while to compare our FLR fluid equations Eqs. (14)-(21) with the previous FLR fluid derivations. For an equilibrium with $p_{\perp}^{(0)}=p_{\|}^{(0)}$, the first four FLR-corrected reduced fluid equations (for the density, parallel velocity, and the perpendicular and parallel pressures) have been derived earlier using both the gyrofluid ${ }^{7}$ and the direct fluid ${ }^{3}$ approaches. Our continuity equation Eq. (14) includes the contributions of the nonlinear polarization flow, and agrees with the equation obtained earlier ${ }^{3,7}$. There has been disagreement between the FLR corrections to the parallel momentum equation obtained from the gyrofluid equations ${ }^{7}$, and those calculated using the fluid theory ${ }^{3}$. Our parallel momentum equation, Eq. (17), agrees with that of the fluid calculations by Smolyakov ${ }^{3}$. As the derivation of the Eq. (17) shows, there is an additional cancellation of the terms proportional to the perturbed perpendicular temperature, when the contributions of the parallel heat fluxes are consistently included. In particular, the term $\sim d^{\prime}\left(\nabla_{\perp}^{2} q_{\|}^{(\perp)}\right) / d t$, which appears when the GF moment is expressed via PF moments: $N V_{\|}=n v_{\|}-\nabla_{\perp}^{2}\|\mu U\| / 2 B$, had been neglected in the previous gyrofluid derivation of parallel momentum balance. However, from Eq. (22), it follows that the contribution from this term is of the same order as other FLR corrections, and it must be retained when $\delta T_{\perp} \neq 0$.

Our particle-fluid equations for the perpendicular and parallel pressure, Eqs. (15) and (16), include the FLR corrections in a form of the "generalized polarization term". Such corrections have been derived previously from the corresponding gyrofluid pressure equations $^{7}$. The FLR-corrected pressure equations obtained in our paper are in general 
agreement with that of the earlier calculation, except for the extra term $\sim p^{2} \nabla_{\perp}^{2} \nabla_{\|} v_{\|}$, which appears in the equation for $p_{\perp}$ in Ref. 7 . This term is cancelled out, when the difference

between the GF and PF parallel heat fluxes $\left(Q_{\|}^{(\perp)}\right.$ and $\left.q_{\|}^{(\perp)}\right)$ is taken into account. From Eq. (10) one has: $\|U \mu\|^{P F}=\|U \mu\|^{G F}+\nabla_{\perp}^{2}\left\|U \mu^{2}\right\| / 2 B$, which combined with relation between $v_{\|}$and $V_{\|}$gives

$$
q_{\|}^{(\perp)}-Q_{\|}^{(\perp)}=\frac{1}{2} \nabla_{\perp}^{2}\left\|U \mu^{2}\right\|-\frac{p_{\perp}}{2 n B} \nabla_{\perp}^{2}\|U \mu\| \approx \frac{p_{\perp}^{2}}{2 n B^{2}} \nabla_{\perp}^{2} v_{\|}
$$

which is correct up to $O(\epsilon)$, and where the higher order moments on the RHS have been neglected.

\section{DERIVATION OF THE GYROVISCOUS FORCE}

\section{A. Parallel component of $\left(\nabla \cdot \pi_{\mathrm{g}}\right)$}

The FLR-corrected parallel momentum equation Eq. (17) can be used to find the parallel component of gyroviscous force. We will define the PF gyroviscosity tensor $\boldsymbol{\pi}_{\mathrm{g}}$ by:

$$
\boldsymbol{\pi}_{\mathrm{g}}=\mathbf{P}-\mathbf{p}^{C G L}-\rho \mathbf{v} \mathbf{v}
$$

where $\mathbf{P}$ is the stress tensor, and $\mathbf{p}^{C G L}=p_{\perp}(\mathbf{I}-\hat{\mathbf{b}} \hat{\mathbf{b}})+p_{\|} \hat{\mathbf{b}} \hat{\mathbf{b}}$ is the particle-fluid ChewGoldberger-Low ${ }^{14}$ (CGL) pressure tensor. Then the parallel component of the fluid momentum equation becomes

$$
n \frac{d v_{\|}}{d t}=-\nabla_{\|} p_{\|}-\left(\nabla \cdot \boldsymbol{\pi}_{\mathrm{g}}\right)_{\|}-n \nabla_{\|} \varphi
$$

To the order considered in this paper, the total time derivative in Eq. (25) can be written as $d / d t=\partial / \partial t+\mathbf{v}_{\perp}^{(1)} \cdot \nabla$, where we have neglected the parallel convective derivative, and used the lowest order expression for the perpendicular fluid velocity, $\mathbf{v}_{\perp}^{(1)}=\mathbf{v}_{E}+\mathbf{v}_{*}$. In this paper 
we define the diamagnetic velocity in terms of $p_{\perp}$ as: $\mathbf{v}_{*}=\hat{\mathbf{b}} \times \nabla p_{\perp} /(n B)$. The comparison of Eqs. (25) and (17), gives the expression for the parallel gyroviscous force in terms of the PF moments

$$
\left(\nabla \cdot \boldsymbol{\pi}_{\mathbf{g}}\right)_{\|}=-\left(n \mathbf{v}_{*} \cdot \nabla\right) v_{\|}+\frac{1}{B^{2}} \nabla_{\perp} \cdot\left\{\nabla_{\perp} \varphi,\|U \mu\|\right\}-\frac{p_{\|}^{(0)}-p_{\perp}^{(0)}}{B^{2}} \nabla_{\|} \nabla_{\perp}^{2} \varphi
$$

The first term in Eq. (26) is responsible for the "gyroviscous cancellation", and the other two are the FLR corrections (the last term on the RHS side can be neglected, when the equilibrium pressure anisotropy is small). Note that our expression for $\left(\nabla \cdot \pi_{\mathbf{g}}\right)_{\|}$does not include the parallel vorticity gradient obtained in previous studies ${ }^{2,12}$. It can be $\operatorname{shown}^{7}$, that this cancels from the parallel momentum equation when other FLR corrections of the same order $O\left(\epsilon^{2}\right)$ are included self-consistently.

\section{B. Perpendicular component of momentum equation}

In the previous Section, we have found the parallel component of momentum equation from the gyrofluid equations up to $O\left(\epsilon^{2}\right)$ and $O\left(\epsilon_{\perp}\right)$. The advantage of the gyrofluid method is that the second-order accurate (in $\epsilon$ ) equations can be obtained from the first-order accurate gyrokinetic equations, which simplifies the calculations considerably. Unfortunately, the perpendicular component of the GF momentum equation cannot be derived in the same way (the GF moment of $\int \mathbf{V}_{\perp} F d U d \mu d \theta$ vanishes, where $\mathbf{V}_{\perp}=B \partial \boldsymbol{\rho} / \partial \theta$ is the particle perpendicular velocity associated with its cyclotron motion). It can be recovered, however, when the PF perpendicular momentum is expressed in terms of the moments of the gyrocenter distribution function $F$ through the second order in $\epsilon$, and expanded in $\epsilon_{\perp}$.

Namely, using the PF momentum equation,

$$
n \frac{d \mathbf{v}_{\perp}}{d t}=-\nabla_{\perp} p_{\perp}-\left(\nabla \cdot \boldsymbol{\pi}_{\mathrm{g}}\right)_{\perp}+n\left(\mathbf{E}_{\perp}+\mathbf{v} \times \mathbf{B}\right)
$$


the perpendicular fluid velocity can be found by expanding in $\epsilon$

$$
\begin{aligned}
\mathbf{v}_{\perp}^{(1)} & =\mathbf{v}_{*}+\mathbf{v}_{E} \\
\mathbf{v}_{\perp} & =\mathbf{v}_{*}+\mathbf{v}_{E}+\underbrace{\frac{1}{n B} \hat{\mathbf{b}} \times\left(\nabla \cdot \boldsymbol{\pi}_{\mathbf{g}}\right)+\frac{1}{B} \hat{\mathbf{b}} \times \frac{d \mathbf{v}_{\perp}^{(1)}}{d t}}_{O\left(\epsilon^{2}\right)}
\end{aligned}
$$

where $d / d t=\partial / \partial t+\mathbf{v}_{\perp}^{(1)} \cdot \nabla$. If the particle-fluid velocity $\mathbf{v}_{\perp}$ is calculated up to $O\left(\epsilon^{2}\right)$, the perpendicular component of gyroviscous force can be found using Eq. (29). In terms of the guiding-center variables the perpendicular momentum can be written $\operatorname{as}^{4}$ :

$$
n \mathbf{v}_{\perp}(\mathbf{x}, t) \equiv \int \mathbf{w}_{\perp} f(\mathbf{w}, \mathbf{x}, t) d^{3} \mathbf{w}=\int \mathbf{V}_{\perp} F_{g c} \delta(\mathbf{X}+\boldsymbol{\rho}-\mathbf{x}) d^{6} \mathbf{Z}
$$

In order to express Eq. (30) in the gyrocenter moments, the relation between the guidingcenter distribution function, $F_{g c}$, and the gyrocenter distribution function, $F$, can be used. This relation is given in the Appendix. Keeping all nonlinear terms through the second order, and expanding in $\epsilon_{\perp}$, we obtain:

$$
\begin{aligned}
n \mathbf{v}_{\perp}= & \frac{1}{B} \hat{\mathbf{b}} \times \nabla P_{\perp}+N \mathbf{v}_{E}+\frac{1}{4 B} \hat{\mathbf{b}} \times \nabla\left(\nabla_{\perp}^{2}\left\|\mu^{2}\right\|\right)-\frac{3}{2 B} \hat{\mathbf{b}} \times \nabla \chi_{\perp} \\
& -\frac{N}{B^{2}}\left(\frac{\partial}{\partial t}+\mathbf{V}_{*} \cdot \nabla\right) \nabla_{\perp} \varphi+\frac{1}{B^{3}} \hat{\mathbf{b}} \times \nabla\left(\nabla_{\perp} P_{\perp} \cdot \nabla_{\perp} \varphi\right)+\frac{1}{2 B^{2}} \mathbf{v}_{E}\left(\nabla_{\perp}^{2} P_{\perp}\right) \\
& +\frac{N}{2 B^{3}} \hat{\mathbf{b}} \times \nabla\left(\nabla_{\perp} \varphi\right)^{2}-\frac{3 P_{\perp}}{4 B^{4}} \nabla_{\perp}^{2} \frac{\partial}{\partial t} \nabla_{\perp} \varphi
\end{aligned}
$$

where the RHS of Eq. (31) is written in terms of the GF moments, and the GF diamagnetic velocity is defined as $\mathbf{V}_{*}=\hat{\mathbf{b}} \times \nabla P_{\perp} /(N B)$, and $\chi_{\perp}=-\left(P_{\perp} / B\right) \hat{\mathbf{b}} \cdot \nabla \times \mathbf{v}_{E}$. Eq. (31) can be used, for example, in kinetic calculations, and it allows one to determine the ion perpendicular current, including FLR and inertia effects, in terms of three GF moments: $N$, $P_{\perp}$ and $\left\|\mu^{2}\right\|$.

To obtain the particle-fluid version of Eq. (31), the GF perpendicular pressure and density on the RHS of Eq. (31) have to be written in terms of the PF moments. First-order 
accurate relation between $N$ and $n$ is sufficient, and it can be obtained from Eq. (11). The perpendicular pressure moment is needed up to $O\left(\epsilon^{2}\right)$, and this can be calculated in the same way as the $\mathbf{v}_{\perp}$ moment in Eq. (30). Thus, we find:

$$
\begin{aligned}
P_{\perp} & =p_{\perp}+\frac{1}{2} n \mathbf{v}_{\perp}^{2}-\frac{1}{2} \nabla_{\perp}^{2}\left\|\mu^{2}\right\|-\frac{2 p_{\perp}}{B^{2}} \nabla_{\perp}^{2} \varphi-\frac{2}{B^{2}} \nabla_{\perp} \varphi \cdot \nabla_{\perp} p_{\perp}-\frac{n}{2 B^{2}}\left(\nabla_{\perp} \varphi\right)^{2} \\
N & =n-\frac{1}{2 B^{2}} \nabla_{\perp}^{2} p_{\perp}-\frac{n}{B^{2}} \nabla_{\perp}^{2} \varphi
\end{aligned}
$$

In terms of particle-fluid moments, neglecting $O\left(\epsilon_{\perp}^{2}\right)$ terms, $n \mathbf{v}_{\perp}$ becomes:

$$
\begin{aligned}
n \mathbf{v}_{\perp}= & n\left(\mathbf{v}_{*}+\mathbf{v}_{E}\right)+\frac{1}{2 B} \hat{\mathbf{b}} \times \nabla\left(\chi_{\perp}-\frac{1}{2} \nabla_{\perp}^{2}\left\|\mu^{2}\right\|+\frac{1}{n B^{2}}\left(\nabla_{\perp} p_{\perp}\right)^{2}\right) \\
- & \frac{n}{B^{2}} \frac{d}{d t} \nabla_{\perp} \varphi-\frac{3 p_{\perp}}{4 B^{4}} \nabla_{\perp}^{2} \frac{\partial}{\partial t} \nabla_{\perp} \varphi
\end{aligned}
$$

Equation (34) includes FLR corrections to the drift velocity, Eq. (28), and nonlinear polarization drifts. The last term represents the FLR correction to the polarization drift velocity. This term is $O\left(\epsilon_{\perp}^{1 / 2}\right)$ order higher than other terms in Eq. (34), but it can be important in some applications (thus, it allows one to obtain the correct dispersion for kinetic Alfven wave $^{15}$ when Eq. (34) is used in two-fluid electromagnetic calculations). For simplicity, this term will be neglected in the subsequent calculations. The divergence of the above expression for the perpendicular momentum, which enters the continuity equation, can be calculated:

$$
\nabla \cdot\left(n \mathbf{v}_{\perp}\right)=\mathbf{v}_{E} \cdot \nabla n-\frac{n}{B^{2}} \frac{d^{\prime}}{d t} \nabla_{\perp}^{2} \varphi+\frac{1}{B^{3}} \nabla_{\perp} \cdot\left\{\nabla_{\perp} \varphi, p_{\perp}\right\}
$$

where we used the relations $\nabla_{\perp} \cdot d^{\prime}\left(\nabla_{\perp} \varphi\right) / d t=d^{\prime}\left(\nabla_{\perp}^{2} \varphi\right) / d t$ and $\nabla_{\perp} \cdot\left(\mathbf{v}_{*} \cdot \nabla\right) \nabla_{\perp} \varphi=-\nabla_{\perp}$. $\left\{\nabla_{\perp} \varphi, p_{\perp}\right\} /\left(n^{(0)} B\right)$. Thus, the continuity equation, Eq. (14), which has been derived from gyrofluid equations, can be recovered again from Eq. (34).

From Eqs. (34) and (29), the perpendicular component of the gyroviscous force can now be found 


$$
\left(\nabla \cdot \boldsymbol{\pi}_{\mathrm{g}}\right)_{\perp}=-n \frac{d \mathbf{v}_{*}}{d t}+\nabla_{\perp} \tilde{\chi}
$$

where $\tilde{\chi}$ is defined as

$$
\tilde{\chi} \equiv \frac{1}{2} \chi_{\perp}-\frac{1}{4} \nabla_{\perp}^{2}\left\|\mu^{2}\right\|+\frac{1}{2 n B^{2}}\left(\nabla_{\perp} p_{\perp}\right)^{2}
$$

The structure of the calculated $\left(\nabla \cdot \pi_{\mathrm{g}}\right)_{\perp}$, Eq. (35), is similar to that obtained by Hazeltine and Meiss ${ }^{12}$ for the $\nabla T=0$ case. The diagonal part of Eq. (35) can be cast in a more familiar form

$$
\tilde{\chi}=-\frac{p_{\perp}}{2 B} \hat{\mathbf{b}} \cdot \nabla \times \mathbf{v}_{\perp}-\frac{1}{4 B} \hat{\mathbf{b}} \cdot \nabla \times \mathbf{q}_{\perp}^{(\perp)}
$$

where the first term is a parallel vorticity term ${ }^{12}$, and the second term appears due to a finite temperature gradient. The component of the perpendicular heat flux is defined by $\mathbf{q}_{\perp}^{(\perp)} \equiv(1 / 2) \int\left(w_{\perp}\right)^{2} \mathbf{w}_{\perp} f d^{3} \mathbf{w}$. In the leading order, it can be shown that

$$
\mathbf{q}_{\perp}^{(\perp)}=B \hat{\mathbf{b}} \times \nabla\left\|\mu^{2}\right\|-2 p_{\perp} \mathbf{v}_{*}=\frac{2 p_{\perp}}{B} \hat{\mathbf{b}} \times \nabla T_{\perp}+\frac{2}{B} \hat{\mathbf{b}} \times \nabla R_{\perp} .
$$

Therefore, when temperature variations (and $\left.R_{\perp}\right)$ are neglected, our expression for $\left(\nabla \cdot \boldsymbol{\pi}_{\mathbf{g}}\right)_{\perp}$ reduces to that of Hazeltine and Meiss ${ }^{12}$.

An alternative expression for the perpendicular gyroviscous force has been derived by several authors ${ }^{2,3}$. The relation between two different forms of gyroviscosity can be found from the equation for the perpendicular pressure including FLR corrections. Thus, using equation (15) for $p_{\perp}$, it can be shown that

$$
\frac{d \mathbf{v}_{*}}{d t}=\left(\mathbf{v}_{*} \cdot \nabla\right) \mathbf{v}_{\perp}-\frac{1}{n} \hat{\mathbf{b}} \times \nabla\left(\nabla_{\|}\|U \mu\|\right)
$$

which allows us to write the perpendicular gyroviscous force in a different form: 


$$
\left(\nabla \cdot \boldsymbol{\pi}_{\mathrm{g}}\right)_{\perp}=-n\left(\mathbf{v}_{*} \cdot \nabla\right) \mathbf{v}_{\perp}+\hat{\mathbf{b}} \times \nabla\left(\nabla_{\|}\|U \mu\|\right)+\nabla_{\perp} \tilde{\chi}
$$

The first term in Eq. (40) is responsible for the gyroviscous cancellation in the perpendicular momentum equation, and other two terms represent off-diagonal and diagonal FLR corrections to the ion stress tensor. Note that previous attempts ${ }^{2,3,12}$ to find a relation between two different representations of the gyroviscous force, Eqs. (35) and (40), failed because they did not account for the pressure anisotropy. Our derivation demonstrates that, to the order which Eq. (39) is valid, both expressions for the perpendicular component of gyroviscous force are equivalent. Substituting Eq. (35) and Eq. (40) into (27), the corresponding two different forms of the FLR corrected ion perpendicular momentum equation can be obtained

$$
\begin{aligned}
& n \frac{d \mathbf{v}_{E}}{d t}=-\nabla_{\perp}\left(p_{\perp}+\tilde{\chi}\right)+n\left(\mathbf{E}_{\perp}+\mathbf{v} \times \mathbf{B}\right) \\
& n \frac{d^{\prime} \mathbf{v}_{\perp}}{d t}=-\nabla_{\perp}\left(p_{\perp}+\tilde{\chi}\right)-\hat{\mathbf{b}} \times \nabla\left(\nabla_{\|}\|U \mu\|\right)+n\left(\mathbf{E}_{\perp}+\mathbf{v} \times \mathbf{B}\right)
\end{aligned}
$$

In previous work, the perpendicular component of the gyroviscous force, including temperature variations, was calculated from the fluid equations by solving the stress tensor evolution equation $^{2,3}$. An expression for $\left(\nabla \cdot \pi_{\mathrm{g}}\right)_{\perp}$, similar in structure to Eq. (40), was obtained. However, in the derivation by Chang and Callen ${ }^{2}$ the contribution of the perpendicular heat flux was neglected, and their expression for $\tilde{\chi}$ is missing a second term proportional to $\nabla T_{\perp}$. As Eq. (38) shows, the perpendicular heat flux is comparable to $\mathbf{v}_{\perp}$ in a plasma with nonuniform temperature. Calculations by Smolyakov ${ }^{3}$ included the heat fluxes and other third-order moments. For the case $\delta T_{\perp}=\delta T_{\|}$, the temperature gradient corrections in Eq. (40) agree with those derived in Ref. 3. Thus, for isotropic temperature, it can be shown that $\mathbf{q}_{\perp}^{(\perp)}=4 / 5 \mathbf{q}_{\perp}$, where $\mathbf{q}_{\perp}=\mathbf{q}_{\perp}^{(\perp)}+\mathbf{q}_{\perp}^{(\|)}$is the total perpendicular heat flux vector. However, in the general case of anisotropic temperature, our results are in partial disagreement with the fluid theory 
results $^{3}$, possibly, because the fluid calculations did not distinguish between the two parts of the perpendicular heat flux, $\mathbf{q}_{\perp}^{(\perp)}$ and $\mathbf{q}_{\perp}^{(\|)}$.

\section{Gyroviscous force in terms of gyrocenter distribution function}

Components of $\left(\nabla \cdot \boldsymbol{\pi}_{\mathrm{g}}\right)$ given by Eqs. (26), (35) and (40) have been calculated in terms of PF moments. In this Section, the expression for the gyroviscous force is given in terms of the GF moments. It is convenient to define the GF gyroviscous stress tensor as

$$
\Pi_{g}=\mathbf{P}-P_{\perp}(\mathbf{I}-\hat{\mathbf{b}} \hat{\mathbf{b}})-P_{\|} \hat{\mathbf{b}} \hat{\mathbf{b}}
$$

where $P_{\perp}=\|\mu B\|$ and $P_{\|}=\left\|U^{2}\right\|$ are GF perpendicular and parallel pressure. In the models relying on kinetic (particle) closure schemes ${ }^{10,16}$, the energetic ion stress tensor is usually approximated by the CGL pressure tensor, $\mathbf{P}^{C G L}=P_{\perp}(\mathbf{I}-\hat{\mathbf{b}} \hat{\mathbf{b}})+P_{\|} \hat{\mathbf{b}} \hat{\mathbf{b}}$, and particle dynamics is described using the drift-kinetic or gyrokinetic approximation. For $k_{\perp} \rho \ll 1$, the next order FLR corrections can be included via the GF gyroviscosity tensor (43). The gyrofluid gyroviscosity tensor, $\boldsymbol{\Pi}_{g}$, is related to PF tensor $\boldsymbol{\pi}_{g}$ by

$$
\boldsymbol{\Pi}_{g}=\boldsymbol{\pi}_{g}+\rho \mathbf{v} \mathbf{v}+\left(p_{\perp}-P_{\perp}-\rho v_{\perp}^{2} / 2\right)(\mathbf{I}-\hat{\mathbf{b}} \hat{\mathbf{b}})+\left(p_{\|}-P_{\|}-\rho v_{\|}^{2}\right) \hat{\mathbf{b}} \hat{\mathbf{b}}
$$

where the difference between PF and GF pressure moments has been included in the definition of $\boldsymbol{\Pi}_{g}$. Expressing particle-fluid moments in terms of gyrocenter-fluid moments, or using Eq. (31) directly, it can be shown:

$$
\begin{aligned}
\left(\nabla \cdot \boldsymbol{\Pi}_{\mathbf{g}}\right)_{\perp} & =N\left(\mathbf{v}_{E} \cdot \nabla\right) \mathbf{V}_{\perp}+\hat{\mathbf{b}} \times \nabla\left(\nabla_{\|}\|\mu U\|\right) \\
& +\nabla_{\perp}\left(\frac{1}{4} \nabla_{\perp}^{2}\left\|\mu^{2}\right\|-\frac{3}{2} \chi_{\perp}+N \mathbf{V}_{*} \cdot \mathbf{v}_{E}\right) \\
\left(\nabla \cdot \boldsymbol{\Pi}_{\mathbf{g}}\right)_{\|} & =N\left(\mathbf{v}_{E} \cdot \nabla\right) V_{\|}+\nabla_{\|}\left(\frac{1}{2 B} \nabla_{\perp}^{2}\left\|U^{2} \mu\right\|-\chi_{\perp}\right)
\end{aligned}
$$




$$
+\frac{1}{B^{2}} \nabla_{\perp} \cdot\left\{\nabla_{\perp} \varphi,\|U \mu\|\right\}+\frac{1}{2 B^{2}}\left\{\varphi, \nabla_{\perp}^{2}\|U \mu\|\right\} / 2
$$

where the RHS is written in terms of GF moments, so that $N V_{\|}=\int U F d U d \mu$, and $\mathbf{V}_{\perp}=$ $\mathbf{v}_{E}+\mathbf{V}_{*}$. Note that the difference between PF and GF pressure moments is of the same order as other FLR corrections, and it must be taken into account in the calculations of $\nabla \cdot \mathbf{P}$

\section{SUMMARY}

In this paper we have presented the derivation of nonlinear gyroviscous forces in a collisionless plasma with temperature variations using gyrokinetic formalism. First, a set of nonlinear gyrofluid equations has been derived in a general form by taking moments of the gyrokinetic Vlasov equation, and expanding with respect to $\epsilon_{\perp}=\left(k_{\perp} \rho\right)^{2}$. A corresponding set of reduced fluid equations is obtained by expressing the gyrocenter-fluid moments in terms of particle-fluid moments. These FLR fluid equations exhibit a generalized form of "gyroviscous cancellation", which appears in an arbitrary high moment equation of an infinite set of fluid equations. The parallel momentum equation, thus obtained, contains FLR corrections up to $O\left(\epsilon_{\perp}\right)$ order in a form of the parallel component of gyroviscous force.

The perpendicular component of the gyroviscous force is found by calculating the perpendicular fluid velocity in terms of gyrocenter-fluid moments and expanding in $\epsilon_{\perp}$. This calculation has been carried out through second-order in the gyrokinetic smallness parameter $\epsilon$, and includes all relevant nonlinear FLR corrections and polarization drifts. Two different forms of the perpendicular gyroviscous force, Eqs. (35) and (40), have been presented, and these are shown to be related by the FLR fluid equation for the perpendicular pressure.

In the derivation of the reduced fluid equations and gyroviscous forces, the FLR cor- 
rections have been systematically accounted for by keeping higher-order moments of the distribution function. It is shown that when the plasma temperature is nonuniform, the contributions of the parallel and perpendicular heat fluxes into the gyroviscous stress tensor are comparable to those of the velocity gradients. The disagreement between the previous fluid and gyrofluid calculations $s^{2,3,7}$ is due to the inconsistent treatment of those heat fluxes.

The gyroviscous forces and reduced fluid equations derived in this paper can be used to include FLR effects into a fluid description of plasma, and serve as a useful benchmark for similar fluid derivations. However, the fluid description, even with the FLR corrections rigorously retained, has to rely on an approximate closure scheme, and generally fails to include other kinetic effects, such as the parallel dynamics and resonant wave-particle interactions. The FLR corrections to the ion stress tensor calculated in terms of the moments of the gyrocenter distribution function represent a systematic FLR expansion, which includes all relevant kinetic effects. Therefore, it can be used in kinetic calculations, for example, to include FLR effects into a drift-kinetic formulation. For the case when $k_{\perp} \rho \ll 1$, such a scheme is more efficient that the gyrokinetic model.

The FLR corrections presented in this paper have been derived assuming a uniform background magnetic field and electrostatic perturbations. These assumptions are commonly used in the derivation of the gyroviscous stress ${ }^{2,3,12}$, and can be justified when the magnetic curvature and finite-beta effects are small and can be accounted for in a perturbative way, for example, in a large aspect ratio tokamak plasma. Since FLR effects are considered as a small correction as well, it is consistent to include the field variation and electromagnetic effects elsewhere in the model. In the general case, however, the magnetic curvature part of the stress tensor and the electromagnetic FLR effects are important, and should be retained. 
The present calculations could be straightforwardly extended to include these effects by using the general geometry electromagnetic gyrokinetic equations ${ }^{4}$. 


\section{ACKNOWLEDGMENTS}

This work was supported by the Department of Energy Contract No. DE-AC02$76 \mathrm{CH} 03037$. 


\section{APPENDIX}

The scalar function $S_{1}$ related to the first order $\left(\right.$ in $\left.\epsilon_{\delta}\right)$ gyrocenter transformation satisfies the equation ${ }^{17,18}$ :

$$
\frac{\partial S_{1}}{\partial t}+U \nabla_{\|} S_{1}+B \frac{\partial S_{1}}{\partial \theta}=\tilde{\varphi}
$$

Expanding the solution up to $O\left(\epsilon \epsilon_{\delta}\right)$, we have $S_{1}=S_{1}^{(1)}+S_{1}^{(2)}$, where

$$
\begin{aligned}
& S_{1}^{(1)}=\frac{1}{B} \tilde{\Psi} \\
& S_{1}^{(2)}=-\frac{1}{B^{2}} \int^{\theta}\left(\frac{\partial \tilde{\Psi}}{\partial t}+U \nabla_{\|} \tilde{\Psi}\right) d \theta^{\prime}
\end{aligned}
$$

For the second order gyrocenter transformation ${ }^{5}$ we have:

$$
\begin{aligned}
B \frac{\partial S_{2}}{\partial \theta}=-\mathbf{G}_{1} \cdot \nabla \bar{\varphi}-G_{1}^{\mu} \frac{\partial \bar{\varphi}}{\partial \mu} & -\frac{1}{2}\left[\left(\mathbf{G}_{1} \cdot \nabla \tilde{\varphi}\right)+G_{1}^{\mu} \frac{\partial \tilde{\varphi}}{\partial \mu}+G_{1}^{\theta} \frac{\partial \varphi}{\partial \theta}\right] \\
+ & \frac{1}{2}\left[\left\langle\mathbf{G}_{1} \cdot \nabla \tilde{\varphi}\right\rangle+\left\langle G_{1}^{\mu} \frac{\partial \tilde{\varphi}}{\partial \mu}\right\rangle+\left\langle G_{1}^{\theta} \frac{\partial \varphi}{\partial \theta}\right\rangle\right]
\end{aligned}
$$

where $\bar{\varphi}=\langle\varphi\rangle$ is the gyroaveraged scalar potential.

In the derivation of the FLR fluid equations (Section III), the first order gyrocenter transformation is sufficient, and the gyrocenter and guiding-center variables are related by: $Z^{j}=Z^{\prime j}+G_{1}^{j}$, where the components of the generating vector can be found using the lowest order solution for $S_{1}=S_{1}^{(1)}$. In this order, the guiding-center distribution function, $F_{g c}$, can be expressed in terms of the gyrocenter distribution, $F$, using the pullback transformation ${ }^{13}$ $F_{g c}=T_{1}^{*}(F)=\exp \left(G_{1}^{j} \partial / \partial Z^{j}\right) F$, so that

$$
F_{g c}=F+\mathbf{G}_{1} \cdot \nabla F+G_{1}^{\mu} \partial F / \partial \mu+G_{1}^{U} \partial F / \partial U
$$

which also follows from: $F_{g c}\left(\mathbf{Z}^{\prime}\right)=F\left(\mathbf{Z}\left(\mathbf{Z}^{\prime}\right)\right)$. 
The calculation of the perpendicular fluid velocity (Section IV) in terms of the gyrocenter-fluid moments requires second order transformation. The guiding-center and the gyrocenter distribution functions are then related by

$$
F_{g c}=F+G_{1}^{j} \frac{\partial F}{\partial Z^{j}}+\frac{1}{2} G_{1}^{j} \frac{\partial}{\partial Z^{j}}\left(G_{1}^{i} \frac{\partial F_{0}}{\partial Z^{i}}\right)+G_{2}^{j} \frac{\partial F_{0}}{\partial Z^{j}}
$$

It is convenient to separate linear and quadratic terms in $\varphi$ (related to the first and second order transformation respectively) in the expression for $F_{g c}$

$$
F_{g c}=F+F^{(1)}+F^{(2)}
$$

where $F^{(1)}=G_{1}^{j} \partial F / \partial Z^{j}$, and $F^{(2)}=\frac{1}{2} G_{1}^{j} \partial\left(G_{1}^{i} \partial F_{0} / \partial Z^{i}\right) / \partial Z^{j}+G_{2}^{j} \partial F_{0} / \partial Z^{j}$. Using the equation for $G_{2}^{\mu}=\partial S_{2} / \partial \theta$, and neglecting the third order terms, the nonlinear part can be simplified:

$$
F^{(2)}=\frac{1}{2}\left(G_{1}^{\mu}\right)^{2} \frac{\partial^{2} F_{0}}{\partial \mu^{2}}-\left(\frac{1}{B} \mathbf{G}_{1} \cdot \nabla \bar{\varphi}+\frac{1}{B} G_{1}^{\mu} \frac{\partial \bar{\varphi}}{\partial \mu}-\frac{1}{2}\left\langle\mathbf{G}_{1} \cdot \nabla G_{1}^{\mu}\right\rangle-\frac{1}{2} \frac{\partial}{\partial \mu}\left\langle\left(G_{1}^{\mu}\right)^{2}\right\rangle\right) \frac{\partial F_{0}}{\partial \mu}
$$

Substituting the expressions for components of the generating vector $G_{1}^{j}$ and $S_{1}$, to the second order in $\epsilon$ and $\epsilon_{\delta}$ :

$$
\begin{aligned}
& F^{(1)}=-\frac{1}{B^{2}} \hat{\mathbf{b}} \times \nabla \tilde{\Psi} \cdot \nabla F+\frac{1}{B} \nabla_{\|} \tilde{\Psi} \frac{\partial F_{0}}{\partial U}+\frac{\tilde{\varphi}}{B} \frac{\partial F}{\partial \mu}-\frac{1}{B^{2}}\left[\frac{\partial \tilde{\Psi}}{\partial t}+U \nabla_{\|} \tilde{\Psi}\right] \frac{\partial F_{0}}{\partial \mu} \\
& F^{(2)}=\frac{\tilde{\varphi}^{2}}{2 B^{2}} \frac{\partial^{2} F_{0}}{\partial \mu^{2}}+\left(\frac{1}{B} \hat{\mathbf{b}} \times \nabla \tilde{\Psi} \cdot \nabla \bar{\varphi}-\tilde{\varphi} \frac{\partial \bar{\varphi}}{\partial \mu}-\frac{1}{2 B}\langle\hat{\mathbf{b}} \times \nabla \tilde{\Psi} \cdot \nabla \tilde{\varphi}\rangle+\frac{1}{2} \frac{\partial}{\partial \mu}\left\langle\tilde{\varphi}^{2}\right\rangle\right) \frac{1}{B^{2}} \frac{\partial F_{0}}{\partial \mu}
\end{aligned}
$$

The last term in the equation for $F^{(1)}$ comes from $S_{1}^{(2)}$, the higher-order in $\omega / \omega_{c i}$ solution for $S_{1}$. It is usually neglected in calculations ${ }^{4,6}$, because $\langle\tilde{\Psi}(\mathbf{x}-\boldsymbol{\rho})\rangle=0$, and its contributions to the density and parallel current integrals are zero. However, it is important to keep this term in the perpendicular current calculations, because it is related to the perpendicular inertia (polarization current) part of $\mathbf{j}_{\perp}$. 
${ }^{1}$ S. I. Braginskii, in Reviews of Plasma Physics, edited by M. A. Leontovich (Consultants Bureau, New York, 1965), Vol. I, p. 205.

2 Z. Chang, and J. D. Callen, Phys. Fluids B 4, 1766 (1992).

${ }^{3}$ A. I. Smolyakov, Can. J. Phys. 76, 321 (1998).

${ }^{4}$ A. Brizard, J. Plasma Physics 41, 541 (1989).

${ }^{5}$ D. H. Dubin, J. A. Krommes, C. Oberman, and W. W. Lee, Phys. Fluids 26, 3524 (1983).

${ }^{6}$ T. S. Hahm, W. W. Lee, and A. Brizard, Phys. Fluids 37, 1940 (1988).

${ }^{7}$ A. Brizard, Phys. Fluids B 4, 1213 (1992).

${ }^{8}$ W. Dorland and G. W. Hammett, Phys. Fluids B 5, 812 (1993).

${ }^{9}$ G. W. Hammett, W. Dorland, and F. W. Perkins, Phys. Fluids B 4, 2052 (1992).

${ }^{10}$ W. Park, E. V. Belova, G. Y. Fu, X. Z. Tang, H. R. Strauss, and L. E. Sugiyama, Phys. Plasmas 6, 1796 (1999).

${ }^{11}$ C. Z. Cheng and J. R. Johnson, J. Geophys. Res. 104, 413 (1999).

${ }^{12}$ R. D. Hazeltine and J. D. Meiss, Phys. Rep. 121, 1 (1985).

${ }^{13}$ R. G. Littlejohn, J. Math. Phys. 23, 742 (1982).

${ }^{14}$ G. F. Chew, M. L. Goldberger, and F. F. Low, Proc. R. Soc. London Ser. A 236, 112 (1956).

${ }^{15}$ A. Hasegawa and L. Chen, Phys. Fluids 19, 1924 (1976). 
${ }^{16}$ C. Z. Cheng, J. Geophys. Res. 96, 21159 (1991).

17 J. R. Cary and R. G. Littlejohn, Ann. Phys. (NY) 151, 1 (1983).

${ }^{18}$ Brizard, A., Nonlinear gyrokinetic tokamak physics, PhD thesis, Princeton University, 1990. 


\section{External Distribution}

Plasma Research Laboratory, Australian National University, Australia

Professor I.R. J ones, Flinders University, Australia

Professor J oão Canalle, Instituto de Fisica DEQ/IF - UERJ , Brazil

Mr. Gerson O. Ludwig, Instituto Nacional de Pesquisas, Brazil

Dr. P.H. Sakanaka, Instituto Fisica, Brazil

The Librarian, Culham Laboratory, England

Library, R61, Rutherford Appleton Laboratory, England

Mrs. S.A. Hutchinson, JET Library, England

Professor M.N. Bussac, Ecole Polytechnique, France

Librarian, Max-Planck-Institut für Plasmaphysik, Germany

J olan Moldvai, Reports Library, MTA KFKI-ATKI, Hungary

Dr. P. Kaw, Institute for Plasma Research, India

Ms. P.J . Pathak, Librarian, Insitute for Plasma Research, India

Ms. Clelia De Palo, Associazione EURATOM-ENEA, I taly

Dr. G. Grosso, Instituto di Fisica del Plasma, Italy

Librarian, Naka Fusion Research Establishment, J AERI, J apan

Library, Plasma Physics Laboratory, Kyoto University, J apan

Research Information Center, National Institute for Fusion Science, J apan

Dr. O. Mitarai, Kyushu Tokai University, J apan

Library, Academia Sinica, Institute of Plasma Physics, People's Republic of China

Shih-Tung Tsai, Institute of Physics, Chinese Academy of Sciences, People's Republic of China

Dr. S. Mirnov, TRINITI, Troitsk, Russian Federation, Russia

Dr. V.S. Strelkov, Kurchatov Institute, Russian Federation, Russia

Professor Peter Lukac, Katedra Fyziky Plazmy MFF UK, Mlynska dolina F-2, Komenskeho Univerzita, SK-842 15 Bratislava, Slovakia

Dr. G.S. Lee, Korea Basic Science Institute, South Korea

Mr. Dennis Bruggink, Fusion Library, University of Wisconsin, USA

Institute for Plasma Research, University of Maryland, USA

Librarian, Fusion Energy Division, Oak Ridge National Laboratory, USA

Librarian, Institute of Fusion Studies, University of Texas, USA

Librarian, Magnetic Fusion Program, Lawrence Livermore National Laboratory, USA

Library, General Atomics, USA

Plasma Physics Group, Fusion Energy Research Program, University of California at San Diego, USA

Plasma Physics Library, Columbia University, USA

Alkesh Punjabi, Center for Fusion Research and Training, Hampton University, USA

Dr. W.M. Stacey, Fusion Research Center, Georgia Institute of Technology, USA

Dr. J ohn Willis, U.S. Department of Energy, Office of Fusion Energy Sciences, USA

Mr. Paul H. Wright, Indianapolis, Indiana, USA 
The Princeton Plasma Physics Laboratory is operated by Princeton University under contract with the U.S. Department of Energy.

\author{
Information Services \\ Princeton Plasma Physics Laboratory \\ P.O. Box 451 \\ Princeton, NJ 08543
}

Phone: 609-243-2750

Fax: 609-243-2751

e-mail: pppl_info@pppl.gov

Internet Address: http://www.pppl.gov 